\title{
Vitamin B12 and Holotranscobalamin Levels in Diabetic Peripheral Neuropathy Patients
}

\author{
${ }^{1}$ Mohammed Noorjahan, ${ }^{2}$ Kandregula Madhavi, ${ }^{3}$ Chandran Priscilla, ${ }^{4}$ Shaik A Jabeen
}

\begin{abstract}
Diabetic neuropathy is the most common and debilitating microvascular complication leading to nontraumatic amputations. Identifying the role of vitamin B12 in the etiology of neuropathy is crucial because simple vitamin B12 replacement may reverse neurologic symptoms if confirmed. Large proportion of vegetarians and patients on metformin have been associated with lowering of vitamin B12 levels. We have undertaken study on 60 vegetarian subjects between the age of 30 and 60 years, 20 healthy controls, 20 type 2 diabetes mellitus patients who were on metformin with peripheral neuropathy (DMWN), and 20 diabetics without peripheral neuropathy (DMWON). Vitamin B12, homocysteine, and folate levels were measured, and holotranscobalamin (holoTC) is also included, to improve the diagnosis of tissue vitamin B12 status, as holoTC is the biologically active fraction and more sensitive index of vitamin B12 status than total vitamin B12 itself. Complete history and clinical evaluation for neuropathy and electroneuromyography were recorded.
\end{abstract}

Results: In the DMWN group, vitamin B12 levels were significantly higher than in the DMWON group and controls [1013 (564-1501) pmol/l, 387 (245-731) pmol/l, and 305 (234-472) pmol// respectively]. Similarly, serum holoTC was also found to be significantly higher in the DMWN [203.8 (129-300) pmol/l] group compared with the DMWON [94.4 (42.2-230.6) pmol/l] group and controls [74 (40-170) pmol/l]. Whereas mean levels of homocysteine and folate did not show any significant difference between groups, significant increase in fasting blood sugar and $\mathrm{HbA} 1 \mathrm{c}$ was seen in the DMWN group compared with DMWON group and controls. Duration of diabetes is also significantly more in DMWN group compared with DMWON group.

Conclusion: We found that vitamin B12 and holoTC levels were high in patients with DMWN and DMWON groups compared with controls. Our study demonstrated that peripheral neuropathy was not associated with vitamin B12 deficiency in diabetics. These findings merit further research on a larger population to investigate into the cause of diabetic neuropathy, the factors involved, and benefit of vitamin B12 supplementation in these patients.

${ }^{1}$ Assistant Professor, ${ }^{2}$ Postgraduate Student, ${ }^{3}$ Professor and Head, ${ }^{4}$ Associate Professor

${ }^{1-4}$ Department of Biochemistry and Neurology, Nizam's Institute of Medical Sciences, Hyderabad, Telangana, India

Corresponding Author: Mohammed Noorjahan, Assistant Professor, Department of Biochemistry and Neurology, Nizam's Institute of Medical Sciences, Hyderabad, Telangana, India Phone: +919440207335, e-mail: m_noorjahan@yahoo.co.in
Key messages: Vitamin B12 status has no association with diabetic peripheral neuropathy.

Keywords: Diabetes mellitus, Holotranscobalamin, Peripheral neuropathy, Vitamin B12.

How to cite this article: Noorjahan M, Madhavi $\mathrm{K}$, Priscilla $\mathrm{C}$, Jabeen SA. Vitamin B12 and Holotranscobalamin Levels in Diabetic Peripheral Neuropathy Patients. Indian J Med Biochem 2016;20(1):16-20.

Source of support: Nil

Conflict of interest: None

\section{INTRODUCTION}

Diabetic neuropathy is the most common and debilitating microvascular complication, with over half of all patients developing altered sensation as a result of damage to peripheral sensory neurons. ${ }^{1}$ It is considered as "silent complication" and is the cause of 50 to $75 \%$ of nontraumatic amputations. ${ }^{2,3}$ It has a profound impact on patients' quality of life and is responsible for majority of diabetes-associated morbidity and mortality. ${ }^{4}$

In India, a country with a large proportion of vegetarians due to cultural and religious beliefs, very high prevalence of vitamin B12 deficiency among the general population has been reported. 5

Metformin has been associated with a lowering of vitamin B12 levels for over 40 years. An increasing number of studies published worldwide during the past decade have further highlighted this correlation, but only one has sought to consider its vitamin B12-lowering effect on neurological function and one other to assess the measurement of vitamin B12 levels in those with peripheral neuropathy receiving metformin therapy. ${ }^{7}$

Identifying the role of B12 in the etiology of neuropathy is crucial because simple vitamin B12 replacement may reverse neurologic symptoms if confirmed. Vitamin B12 measurements are not always reflective of decreased vitamin B12 status since patients with clinically defined vitamin B12 deficiency do not always have low serum vitamin B12 concentrations. ${ }^{8-10}$ Conversely, many patients with mildly reduced vitamin B12 levels have no evidence of clinical deficiency. It is recognized that holotranscobalamin (holoTC), the biologically active fraction of vitamin B12 (also known as active B12), is a more sensitive index of vitamin B12 status than the 
measurement of total vitamin B12 itself. $^{11}$ Vitamin B12 deficiency is traditionally diagnosed by laboratory findings of low serum vitamin B12 levels, typically in the setting of megaloblastic anemia. However, subclinical vitamin B12 deficiency often presents with normal serum vitamin B12. ${ }^{12}$ We have used more specific diagnostic marker holoTC, homocysteine, and folate levels along with serum total vitamin B12 levels to improve the diagnosis of tissue vitamin B12 status in the diabetic population who are suffering from diabetic peripheral neuropathy.

\section{MATERIALS AND METHODS}

This is a cross-sectional observational study carried out in a tertiary care hospital. Study included 60 subjects between the age group of 30 to 60 years, 20 healthy controls, 20 known type 2 diabetes mellitus patients with peripheral neuropathy (DMWN), and 20 type 2 diabetes mellitus patients without peripheral neuropathy (DMWON). All the study subjects were vegetarians. Patients were excluded if they had been on metformin for less than 6 months or if they had a history of alcoholism, smoking or other drug abuse, psychiatric disease, pernicious anemia, stomach or bowel disease, bowel surgery, chronic renal failure or liver disease, and also nonvegetarians.

\section{Clinical Assessment}

Medical history was recorded and physical examination was undertaken. Body mass index (BMI) was calculated from height and weight measurements. Peripheral neuropathy was diagnosed according to clinical, electroneuromyography, and autonomic function testing.

\section{Biochemical Investigations}

Fasting blood samples were obtained for estimation of plasma glucose (by hexokinase method), glycated hemoglobin (HbA1c by high-performance liquid chromatography), serum vitamin B12, serum folate, and plasma homocysteine (by electrochemiluminescence method). Separated serum is stored at $-20^{\circ} \mathrm{C}$ for the analysis of holoTC (by enzyme-linked immunosorbent assay method).

\section{Statistical Analysis}

Descriptive statistics of normally distributed variables is reported as mean and SD and that of non-normally distributed variables is reported as median, 25th, to 75th percentile. To test the differences between two groups, i.e., cases and controls, we used unpaired Student's t-test for normally distributed variables and Mann-Whitney $\mathrm{U}$ test for non-normally distributed variables; $\mathrm{p}<0.05$ was considered as statistically significant.

\section{RESULTS}

\section{Baseline Characteristics}

Study included a total of 60 subjects that included 40 type 2 diabetes mellitus patients and 20 healthy controls. Out of 40 , a total of 20 diabetics with peripheral neuropathy were included in the DMWN group (15 men and 5 women) and 20 diabetic patients without peripheral neuropathy in the DMWON group (9 men and 11 women).

The mean age was $54 \pm 6.1$ years in the DMWN group, $50.8 \pm 6.7$ years in the DMWON group and $51.2 \pm 6.0$ years in controls. Hemoglobin A1c was $8.8 \pm 2.5 \%$ in the DMWN group and $7.7 \pm 1.6 \%$ in the DMWON group. All the groups' BMI was comparable. Baseline characteristics are shown in Table 1.

The mean duration of diabetes was $10 \pm 1.6$ and $6.4 \pm 1.7$ years in DMWN and DMWON groups respectively, which was significant. Fasting blood sugar (FBS) also has shown statistically significant increase in DMWN group when compared with DMWON group and control group.

\section{Marker of Vitamin B12 Status}

In the DMWN group [1013 (564-1501) pmol/L], the serum vitamin B12 levels were significantly higher than in the

Table 1: Demographic variables in the study groups

\begin{tabular}{llll}
\hline Variable & $D M W N(n=20)$ & $D M W O N(n=20)$ & Contro/s $(n=20)$ \\
\hline Age $(\mathrm{yrs})$ & $54 \pm 6.1$ & $50.8 \pm 6.7$ & $51.2 \pm 6$ \\
BMI $\left(\mathrm{kg} / \mathrm{m}^{2}\right)$ & $27.9 \pm 5.6$ & $27.6 \pm 5.4$ & $26.5 \pm 5.5$ \\
Duration of DM $(\mathrm{yrs})$ & $10 \pm 1.6^{*}$ & $6.4 \pm 1.7$ & - \\
$\mathrm{FBS}(\mathrm{mg} / \mathrm{dL})$ & $184.5 \pm 85^{\star \ddagger}$ & $138 \pm 55^{\ddagger}$ & $89 \pm 25$ \\
$\mathrm{HbA} 1 \mathrm{c}(\%)$ & $8.8 \pm 2.5^{\ddagger}$ & $7.7 \pm 1.6^{\ddagger}$ & $5.4 \pm 0.4$ \\
Folate $(\mathrm{ng} / \mathrm{mL})$ & $12 \pm 7.7$ & $12.3 \pm 6$ & $10.5 \pm 4.8$ \\
Homocysteine $(\mu \mathrm{mol} / \mathrm{L})$ & $12.8 \pm 2.9$ & $10.9 \pm 5.6$ & $9.5 \pm 4.5$ \\
Vitamin B12 $(\mathrm{pmol} / \mathrm{L})$ & $1013(564-1501)^{\star \ddagger}$ & $387(245-731)^{\dagger}$ & $305(234-472)$ \\
Holotranscobalamin $(\mathrm{pmol} / \mathrm{L})$ & $203.8(129-300)^{\star \ddagger}$ & $94.4(42.2-230.6)^{\dagger}$ & $74(40-170)$ \\
\hline${ }^{*} \mathrm{p} \leq 0.05$ is taken as significant between DM groups; ${ }^{\dagger} \mathrm{p} \leq 0.05$ is taken as significant between DM group and controls; ${ }^{\ddagger} \mathrm{p}<0.001$ \\
between DM and controls
\end{tabular}




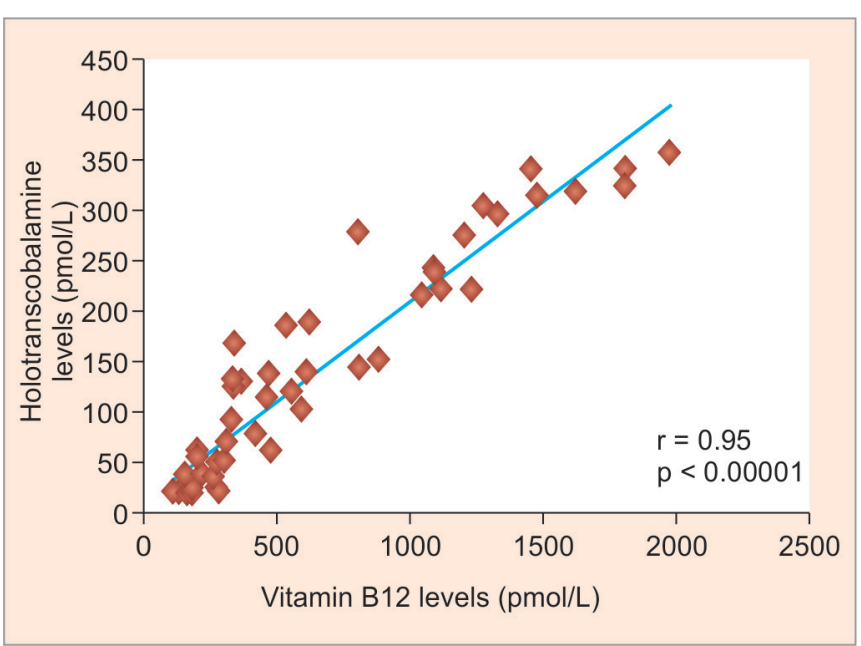

Graph 1: Correlation between vitamin B12 and holoTC

DMWON group [387 (245-731) pmol/L] and control group [305 (234-472) pmol/L] (Table 1). Similarly serum holoTC also showed significantly higher levels in the DMWN group [203.8 (129-300) pmol/L] compared with DMWON group [94.4 (42.2-230.6) pmol/L] and control group [74 (40-170) pmol/L].

As shown in Graph 1, serum vitamin B12 and holoTC have a very good correlation $(\mathrm{r}=0.95, \mathrm{p}<0.00001)$. Although markers of vitamin B12 status differed between the groups, it was within the normal range or high in both the DMWN and DMWON groups compared with controls. Holotranscobalamin has not added any additional information on vitamin B12 status of the patient.

Mean levels of plasma homocysteine did not show any statistically significant difference between the groups, which was $12.8 \pm 2.9 \mu \mathrm{mol} / \mathrm{L}$ in the DMWN group, $10.9 \pm 5.6 \mu \mathrm{mol} / \mathrm{L}$ in the DMWON group, and $9.5 \pm 4.5$ $\mu \mathrm{mol} / \mathrm{L}$ in controls. Mean levels of folate also did not show any statistical difference between the groups (Table 1).

The significance of difference for all the variables between the groups was compared by using unpaired Student's t-test. There was no significant difference between the mean levels of age $(p=0.10)$, BMI $(p=0.84)$, HbA1c $(p=0.13)$, folate $(p=0.69)$, and homocysteine $(p=0.34)$ among the diabetic groups. Duration of diabetes $(p=0.04)$ and FBS $(p=0.04)$ showed significant difference among the two diabetic groups. Contrary to the expectations, total vitamin B12 and holoTC were significantly higher in DMWN group compared with DMWON group and controls.

\section{DISCUSSION}

Diabetic peripheral neuropathy is a common disorder and is defined as signs and symptoms of peripheral nerve dysfunction in a patient with diabetes mellitus in whom other causes of peripheral nerve dysfunction have been excluded. ${ }^{13}$ It is a collection of syndromes, either focal or diffuse in nature, affecting sensory, motor, and/ or autonomic peripheral neurons. ${ }^{14,15}$ These disorders can range from clinical to subclinical and differ in their anatomical distribution, clinical course, and spectrum of symptoms. ${ }^{1}$

The mechanisms underlying the development of these dichotomous symptoms remain a significant question. A number of large studies have clearly identified hyperglycemia as a key feature involved in the pathogenesis of diabetic neuropathy. ${ }^{16-19}$ This supports our results, which showed significant increase in FBS in DMWN group compared with DMWON group $(p=0.046)$ and in comparison with controls $(p=0.0001$ for DMWN and $p=0.0008$ for DMWON). Poor glycemic control has been recognized as an important risk factor for the development and progression of neurological complications. Glycated hemoglobin levels also showed increase in both the DM groups compared with controls. The Diabetes Control and Complications Trial (DCCT) study found that intensive therapy reduced the risk of developing confirmed clinical neuropathy by $64 \%$ compared with conventional therapy after an average follow-up of 6.5 years. ${ }^{20}$

Duration of diabetes also showed statistically significant increase $(\mathrm{p}<0.0001)$ in DMWN group compared with DMWON group. Similarly, the EURODIAB study found both duration of diabetes and quality of glycemic control as risk factors for the development of diabetic neuropathy. ${ }^{19}$ Thus, prior exposure to hyperglycemia predisposes to greater risk of developing diabetic peripheral neuropathy. ${ }^{1}$

Due to hyperglycemia, glucose levels rose within sensory neurons and normal metabolic pathways became overwhelmed and excess glucose was shunted into other ancillary pathways that, under these conditions, became damaging. One consequence of hyperglycemia is the increased and accelerated production of advanced glycation end products (AGEs) in tissues where damage results in secondary complications involving peripheral nerves. Advanced glycation end products have been shown to play a major role in the pathogenesis of diabetic neuropathy. ${ }^{1}$

Neurological markers like serum vitamin B12, homocysteine, and folate levels were within normal ranges in both the diabetic groups. Our results are in par with a study by Russo et al. ${ }^{21}$ Metformin therapy is associated with a mild vitamin B12 level reduction, but not with diabetic peripheral neuropathy.

Of note, only two case reports have been published showing metformin-related B12 deficiency presenting as a peripheral neuropathy. ${ }^{22}$ In light of such reports, it has 
been proposed that annual vitamin B12 supplementation should be given to such patients receiving metformin therapy, ${ }^{23}$ and indeed, in certain parts of the world this has become a part of recognized empirical practice. ${ }^{24}$ The high levels of total vitamin B12 and holoTC in DMWN patients in our study may be attributed to this practice. This explains that diabetic neuropathy may occur irrespective of vitamin B12 status. Holotranscobalamin has shown good correlation with vitamin B12 showing adequate tissue levels of vitamin B12 in these patients. Our results suggest that factors involved in maintaining an adequate vitamin B12 status are not protective against neuropathy.

High plasma levels of folate and especially vitamin B12 may even have a detrimental effect. In fact, Hultdin et $\mathrm{al}^{25}$ study has shown that increasing plasma vitamin B12 was associated with an up to 3-fold increase in prostate cancer risk, which was independent of age, follow-up time, and disease state at diagnosis. In another study, vitamin B12 has been implicated in genotoxicity in smokers, ${ }^{26}$ and some preliminary evidence suggests a possible increased risk of lung cancer with increasing plasma vitamin B12 levels. ${ }^{27,28}$

Our study has limitation due to the small sample size; its statistical power might be insufficient.

Our future consideration is to examine AGE levels that may play a role in the occurrence of diabetic neuropathy independent of vitamin B12 levels in this group of patients.

\section{CONCLUSION}

In the DMWN group, serum vitamin B12 and holoTC levels were significantly higher than in the DMWON and control groups, making the decision of vitamin B12 supplementation a complex one. There was no significant difference between the mean levels of age, $\mathrm{BMI}, \mathrm{HbA} 1 \mathrm{c}$, folate, and homocysteine among the diabetic groups. Duration of diabetes and FBS showed significant difference among the two diabetic groups. Contrary to expectations, total vitamin B12 and holoTC were significantly higher in the DMWN group compared with DMWON group and controls. Our study demonstrated that peripheral neuropathy was not associated with serum vitamin B12 deficiency in diabetics. These findings merit further research on a larger population using additional markers to investigate the cause of diabetic neuropathy, the factors involved, and benefit of vitamin B12 supplementation in these patients.

\section{REFERENCES}

1. Jack M, Wright D. Role of advanced glycation end products and glyoxalase I in diabetic peripheral sensory neuropathy. Transl Res 2012 May;159(5):355-365.
2. Holzer SE, Camerota A, Martens L, Cuerdon T, CrystalPeters J, Zagari M. Costs and duration of care for lower extremity ulcers in patients with diabetes. Clin Ther 1998 Jan-Feb;20(1):169-181.

3. Caputo GM, Cavanagh PR, Ulbrecht JS, Gibbons GW, Karchmer AW. Assessment and management of foot disease in patients with diabetes. N Engl J Med 1994 Sep;331(13): 854-860.

4. Vinik EJ, Hayes RP, Oglesby A, Bastyr E, Barlow P, FordMolvik SL, Vinik AI. The development and validation of the Norfolk QOL-DN, a new measure of patients' perception of the effects of diabetes and diabetic neuropathy. Diabetes Technol Ther 2005 Jun;7(3):497-508.

5. Yajnik C, Deshpande S, Lubree HG, Naik SS, Bhat DS, Uradey BS, Deshpande JA, Rege SS, Refsum H, Yudkin JS. Vitamin B12 deficiency and hyperhomocysteinemia in rural and urban Indians. J Assoc Physicians India 2006 Oct;54:775-782.

6. Shobhaa V, Tareya S, Singh RG, Shetty P, Unni US, Srinivasan K, Kurpad AV. Vitamin B12 deficiency and levels of metabolites in an apparently normal urban south Indian elderly population. Indian J Med Res 2011 Oct;134:432-439.

7. Wile DJ, Toth C. Association of metformin, elevated homocystein and methylmalonic acid levels and clinically worsened diabetic peripheral neuropathy. Diabetic Care 2010 Jan;33(1):156-161.

8. Clarke R, Refsum H, Birks J, Evans JG, Johnston C, Sherliker P, Ueland PM, Schneede J. PcPartlin J, Nexo E, et al. Screening for vitamin B-12 and folate deficiency in older persons. Am J Clin Nutr 2003 May;77(5):1241-1247.

9. Lindenbaum J, Savage DG, Stabler SP, Allen RH. Diagnosis of cobalamin deficiency. II. Relative sensitivities of serum cobalamin, methylmalonic acid, and total homocysteine concentrations. Am J Hematol 1990 Jun;34(2):99-107.

10. Wald NJ, Law M, Hoffbrand VA. Vitamin B-12 and folate deficiency in elderly persons. Am J Clin Nutr 2004 Feb;79(2):338.

11. Hvas AM, Nexo E. Holotranscobalamin - a first choice assay for diagnosing early vitamin B12 deficiency? J Intern Med 2005 Mar;257(3):289-298.

12. Snow CF. Laboratory diagnosis of vitamin B12 and folate deficiency: a guide for the primary care physician. Arch Intern Med 1999 Jun;159(12):1289-1298.

13. Bansal V, Kalita J, Misra UK. Diabetic neuropathy. Postgrad Med J 2006 Feb;82(964):95-100.

14. Vinik AI, Park TS, Stansberry KB, Pittenger GL. Diabetic neuropathies. Diabetologia 2000 Aug;43(8):957-973.

15. Edwards JL, Vincent AM, Cheng HT, Feldman EL. Diabetic neuropathy: mechanisms to management. Pharmacol Ther 2008 Oct;120(1):1-34.

16. DCCT. The effect of intensive treatment of diabetes on the development and progression of long-term complications in insulin-dependent diabetes mellitus. The Diabetes Control and Complications Trial Research Group. N Engl J Med 1993 Sep;329:977-986.

17. Pop-Busui R, Herman WH, Feldman EL, Low PA, Martin CL, Cleary PA, Waberski BH, Lachin JM, Albers JW; DCCT/EDIC Research Group. DCCT and EDIC studies in type 1 diabetes: lessons for diabetic neuropathy regarding metabolic memory and natural history. Curr Diab Rep 2010 Aug;10(4):276-282.

18. Albers JW, Herman WH, Pop-Busui R, Feldman EL, Martin CL, Cleary PA, Waberski BH, Lachin JM; Diabetes Control and Complications Trial/Epidemiology of Diabetes 
Interventions and Complications Research Group. Effect of prior intensive insulin treatment during the Diabetes Control and Complications Trial (DCCT) on peripheral neuropathy in type 1 diabetes during the Epidemiology of Diabetes Interventions and Complications (EDIC) Study. Diabetes Care 2010 May;33(5):1090-1096.

19. Tesfaye S, Stevens LK, Stephenson JM, Fuller JH, Plater M, Ionescu-Tirgoviste C, Nuber A, Pozza G, Ward JD. Prevalence of diabetic peripheral neuropathy and its relation to glycaemic control and potential risk factors: the EURODIAB IDDM Complications study. Diabetologia 1996 Nov;39(11):1377-1384.

20. The Diabetes Control and Complications Trial Research Group. The effect of intensive diabetes therapy on the development and progression of neuropathy. Ann Intern Med 1995 Apr;122(8):561-568.

21. Russo GT, Giandalia A, Romeo EL, Scarcella C, Gambadoro N, Zingale R, Forte F, Perdichizzi G, Alibrandi A, Cucinotta D. Diabetic neuropathy is not associated with homocysteine, folate, vitamin B12 levels, and MTHFR C677T mutation in type 2 diabetic outpatients taking metformin. J Endocrinol Invest 2016 Mar;39(3):305-314.

22. Bell DS. Metformin-induced vitamin B12 deficiency presenting as a peripheral neuropathy. South Med J 2010 Mar;103(3): 265-267.
23. Mahajan R, Gupta K. Revisiting metformin: annual vitamin B12 supplementation may become mandatory with long-term metformin use. J Young Pharm 2010 Oct;2(4):428-429.

24. Chen S, Lansdown AJ, MoatSJ, Ellis R, Goringe A, Dunstan FDJ, Rees JAE. An observational study of the effect of metformin on B12 status and peripheral neuropathy. Br J Diabetes Vasc Dis 2012 Aug;12:189.

25. Hultdin J, Van Guelpen B, Bergh A, Hallmans G, Stattin P. Plasma folate, vitamin B12, and homocysteine and prostate cancer risk: a prospective study. Int J Cancer 2005 Feb 20;113:819-824.

26. Zijno A, Andreoli C, Leopardi P, Marcon F, Rossi S, Caiola S, Verdina A, Galati R, Cafolla A, Crebelli R. Folate status, metabolic genotype, and biomarkers of genotoxicity in healthy subjects. Carcinogenesis 2003 Jun;24(6):1097-1103.

27. Gurdal-Yuksel E, Karadag M, Ozyardimci N, Kunt-Uzaslan AE, Yarkin T. Cigarette smoking, serum lipids, folate, and vitamin B12 in lung cancer. J Environ Pathol Toxicol Oncol 1996;15(2-4): 161-167.

28. Hartman TJ, Woodson K, Stolzenberg-Solomon R, Virtamo J, Selhub J, Barrett MJ, Albanes D. Association of the B-vitamins pyridoxal 5 -phosphate $(\mathrm{B}(6)), \mathrm{B}(12)$, and folate with lung cancer risk in older men. Am J Epidemiol 2001 Apr 1;153(7):688-694. 